\title{
Tarifregulierung zwischen Fläche und Betrieb: Koordinierung und Praxis in der Chemie- und der Metallindustrie
}

Thomas Haipeter

\begin{abstract}
Dezentralisierung ist einer der zentralen Trends des deutschen Tarifsystems. Die bislang weitgehendste Form der Dezentralisierung stellen Tarifabweichungen dar. Sie zeichnen sich durch zwei Merkmale aus: Standards der Flächentarifverträge werden betrieblich unterschritten, und diese Unterschreitungen werden legitimiert. Anders als wilde Tarifunterschreitungen finden sie mit ausdrücklicher Billigung der Tarifvertragsparteien statt und werden vielfach auch von ihnen mitverhandelt. Über die Auswirkungen der Tarifabweichungen auf das System der Flächentarifverträge ist wenig bekannt. In diesem Artikel sollen am Beispiel zweier Schlüsselbranchen der Tarifabweichung, der Metallindustrie und der chemischen Industrie, empirische Entwicklungen und Probleme der Tarifabweichung aufgezeigt werden.
\end{abstract}

\section{Tarifabweichung: Kontrol- lierte Dezentralisierung?}

Seit Mitte der 1990er Jahre sind in so unterschiedlichen Branchen wie der chemischen Industrie, dem Einzelhandel, der Bauindustrie oder der Metallindustrie von den Tarifvertragsparteien Möglichkeiten zur Tarifabweichung vereinbart worden (vgl. für einen Überblick Bispinck 2004a). Sie tragen in den Flächentarifverträgen unterschiedliche Bezeichnungen wie Härtefallklauseln, Sanierungsklauseln, Öffnungsklauseln oder Flexibilisierungsinstrumente. Allen diesen Varianten ist gemeinsam, dass es um betriebliche Unterschreitungen flächentarifvertraglicher Standards der Arbeits- und Einkommensbedingungen geht, die durch entsprechende Regelungen in den Flächentarifverträgen legitimiert werden. Zumeist werden die Abweichungen auch von den oder mit Beteiligung der Tarifvertragsparteien ausgehandelt oder müssen zumindest von ihnen genehmigt werden. Eine vollständige Verlagerung der Regulierungsverantwortung auf die Betriebsparteien findet sich nur in wenigen und inhaltlich eng definierten Fällen (so im letzten Lohntarifvertrag der Metallindustrie vom November 2008 die Verschiebung der zweiten Stufe der Lohnerhöhung).

Unabhängig von ihrer konkreten Regelung aber sind Tarifabweichungen zunächst per se eine grundlegende Herausforderung für die Prägekraft der Flächentarifvertragsnormen. Nicht zuletzt auch deshalb sind ihre Auswirkungen in der Literatur so um- stritten. Die Interpretationen reichen von der Einschätzung, Tarifabweichungen seien gelungene Formen der Anpassung kollektivvertraglicher Regulierungen an gewandelte wirtschaftliche Bedingungen (Jacobi 2003) bis hin zu der Bewertung, sie seien Triebkräfte der Erosion des Tarifsystems (Hassel 1999). Empirische Analysen der Tarifabweichung stehen jedoch noch aus. Diese Forschungslücke soll im Folgenden durch die Analyse der Entwicklung und Praxis der Tarifabweichung in der chemischen Industrie und der Metallindustrie bearbeitet werden. Auf dieser Grundlage lassen sich dann empirisch gehaltvolle Aussagen zur Frage der Auswirkungen der Tarifabweichung auf die Flächentarifverträge und die Prägekraft ihrer Normen treffen. ${ }^{1}$

Chemische Industrie und Metallindustrie sind nicht nur traditionell Schlüsselbranchen des Tarifvertragssystems, sie weisen auch jeweils besonders weitreichende und dabei in der Form unterschiedliche Regelungen zur Tarifabweichung auf. Während in der chemischen Industrie die Tarifabweichung als gemeinsames Projekt der Tarifparteien zur Stärkung des Flächentarifvertrages verstanden wird, ist sie in der Metallindustrie Ergebnis langanhaltender Konflikte, äußeren politischen Drucks und wachsender Probleme der betrieblichen Abweichungspraxis (dazu auch Bispinck 2004b). Und während in der chemischen Industrie Abweichungen als Betriebsvereinbarungen mit Zustimmungspflicht der Tarifparteien gestaltet werden, haben sie in der Metallindustrie den Status von betriebsbezogenen Ergänzungstarifverträgen.

\subsection{LEGITIMIERTE TARIFUNTERSCHREITUNGEN}

Tarifabweichungen sind ihrer Form nach betriebliche Beschäftigungsbündnisse. Wie diese stellen sie "Tauschpakete“ (MassaWirth 2007) mit der Zielkombination aus Beschäftigungssicherung und Verbesserung der Wettbewerbsfähigkeit dar. Auch sind sie, wie betriebliche Bündnisse, dadurch charakterisiert, dass sie eine Vielzahl originär tariflicher Regelungsgegenstände behandeln können (Seifert/Massa-Wirth 2005). Zwei Spezifika allerdings unterscheiden die Tarifabweichungen von betrieblichen Bündnissen: Sie legen Unterschreitungen von Tarifnormen fest, und diese Unterschreitungen sind von den Tarifvertragsparteien legitimiert. Während betriebliche Bündnisse ansonsten übertarifliche Leistungen der Unternehmen oder tariflich nicht geregelte Themen zum Gegenstand haben, werden bei Tarifabweichungen Flächentarifvertragsnormen durch niedrigere betriebliche Arbeitsstandards zumindest temporär ersetzt. Dadurch entsteht eine mehr oder weniger

\footnotetext{
Grundlage meiner Darstellung ist ein von der Hans-Böckler-Stiftung gefördertes Projekt zum Wandel der Mitbestimmung bei Tarifabweichungen in der chemischen Industrie und der Metallindustrie.
}

Thomas Haipeter, Dr., Institut Arbeit und Qualifikation (IAQ), Universität DuisburgEssen. Arbeitsschwerpunkte: Industrielle Beziehungen, Industriesoziologie. e-mail: thomas.haipeter@uni-due.de 
offene Normkonkurrenz der Arbeitsstandards - mit zunächst uneindeutigen Folgen hinsichtlich der Prägekraft der Flächentarifverträge.

Aufgrund ihrer Legitimierung seitens der Tarifvertragsparteien unterscheiden sich Tarifabweichungen von informellen Unterschreitungen der Tarifnormen. Diese wilde Dezentralisierung kann ebenfalls im Rahmen betrieblicher Bündnisse vorkommen (Rehder 2003), sie kann aber auch den Charakter eines reinen Concession Bargaining ohne Gegenleistungen der Unternehmen haben. Für die wilde Dezentralisierung fällt die Diagnose ihrer Auswirkungen auf die Flächentarifverträge eindeutig aus: Sie bedeutet eine Erosion der Tarifvertragsnormen. Tarifnormen verlieren ihre Prägekraft für die unterschreitenden Betriebe, und es gibt keine kollektiven Akteure, die auf Wiedereinhaltung der Tarifnormen drängen würden. Bei der Tarifabweichung hingegen wird die Unterschreitung von den Tarifvertragsparteien offiziell gebilligt und in den meisten Fällen auch aktiv umgesetzt. Die entscheidende empirische Frage ist daher, ob dies für die Prägekraft der Flächentarifverträge einen Unterschied macht.

\subsection{INHALTLICHE UND PROZEDURALE KONTROLLE ALS PROBLEM DER TARIFABWEICHUNG}

Die Verbindung von Tarifunterschreitung und Legitimation legt es auf den ersten Blick nahe, die Tarifabweichung als kontrollierte oder organisierte Dezentralisierung zu bezeichnen (Traxler 1995). Denn in der Kontrolle scheint der entscheidende Unterschied zur wilden Dezentralisierung zu bestehen. Die Kontrolle der Tarifabweichung ist jedoch keine Selbstverständlichkeit, die sich automatisch aus der Existenz entsprechender Regelungsnormen im Tarifvertrag ergibt. Ob die Tarifabweichung tatsächlich ein kontrollierter Prozess ist, kann erst nach Untersuchung der damit verbundenen sozialen Praktiken und ihrer materiellen Ergebnisse beurteilt werden. Sie entsteht nur als Ergebnis einer aktiven Ordnungsleistung der Tarifakteure. Besteht eine solche Kontrolle nicht, wäre in den Auswirkungen kaum ein Unterschied zwischen der Tarifabweichung und der wilden Dezentralisierung festzustellen.

Die Kontrolle der Tarifabweichung ist in zwei Richtungen zu untersuchen. Die erste Richtung ist die inhaltliche Kontrolle.
Damit ist die Kontrolle der Anzahl der Tarifabweichungen und ihrer Inhalte gemeint. Je seltener Tarifabweichungen abgeschlossen werden und je geringer die damit verbundenen materiellen Konzessionen im Vergleich zu den Flächentarifvertragsstandards sind, umso eher kann von einer kontrollierten Dezentralisierung gesprochen werden und umso geringer sind die negativen Auswirkungen der Tarifabweichungen auf die Prägekraft der Flächentarifvertragsnormen. Auch können Gegenleistungen der Unternehmen, wie Beschäftigungsund Standortsicherung oder Investitionszusagen, die materiellen Konzessionen abfedern und zumindest teilweise aufwiegen; zugleich können sie die Perspektive einer Rückkehr zu den Flächentarifvertragsnormen beinhalten.

Die zweite Richtung der Analyse ist die Prozesskontrolle. Sie bezieht sich auf das Problem der Artikulation (Crouch 1993), also die Frage, wie in den Verbandsorganisationen Zuständigkeiten und Kommunikationswege zwischen den Organisationsebenen gestaltet werden. Prozesskontrolle umfasst sowohl die Transparenz von Verfahren und die Entscheidung über die Einleitung von Verfahren - also die Bewertung der Ausgangsbedingungen in den Unternehmen - als auch die Kontrolle der Verhandlungsdynamik und ihrer Ergebnisse. Dazu gehört auch die Unterbindung der Konkurrenz über Arbeitskosten, die zwischen unterschiedlichen Unternehmen einer Branche oder zwischen unterschiedlichen Standorten in einem Konzern durch Tarifabweichungen droht und die zu einem „Race to the Bottom“ der Arbeitsstandards führen kann.

Tarifabweichungen sind eine Folge des - zumindest relativen - Rückgangs der gewerkschaftlichen Organisations- und Verhandlungsmacht. Ursächlich hierfür ist vor allem - abgesehen vom Mitgliederverlust der Gewerkschaften - der Zugewinn an Verhandlungsmacht, den die Unternehmen im Zuge von Globalisierung und Finanzialisierung gegenüber den Gewerkschaften - und übrigens auch gegenüber ihren Arbeitgeberverbänden - realisieren können. Die Globalisierung der Produktion ermöglicht ihnen, durch die Entfachung von Standortkonkurrenz Druck auf Einkommensstandards und Arbeitsbedingungen auszuüben. Und die Orientierung der Unternehmensgovernance an Normen und Erwartungen der Finanzmarktakteure wiederum legitimiert die Steigerung der
Renditeerwartungen und die Auslagerung von Geschäftsfeldern und Betrieben, die diesen Erwartungen nicht gerecht werden. Daher überrascht es nicht, dass die Initiative zur Tarifabweichung bei den Unternehmen liegt, sei es in Form einer konkreten Forderung nach Tarifunterschreitungen oder nur in Form eines allgemeinen Drohszenarios. Die Gewerkschaften befinden sich in einer defensiven Position, die ihnen die Kontrolle von Tarifabweichungen naturgemäß erschwert.

\section{Tarifliche Regelungen zur Tarifabweichung in den Branchen}

In den beiden Untersuchungsbranchen wurden bereits in der ersten Hälfte der 1990er Jahre Regelungen zur Tarifabweichung in den Flächentarifverträgen verankert, und in beiden Branchen wurden diese Regelungen schrittweise thematisch und hinsichtlich ihrer Bedingungen und Geltungsbereiche ausgeweitet. Dies geschah jedoch unter sehr unterschiedlichen tarifpolitischen Vorzeichen.

\subsection{CHEMISCHE INDUSTRIE}

In den Tarifverträgen der chemischen Industrie existieren mehrere Regelungen zur Tarifabweichung. Sie werden von den Tarifparteien selber als Öffnungsklauseln oder Flexibilisierungsinstrumente bezeichnet und als tarifpolitische Innovationen betrachtet, die die Flexibilisierungsmöglichkeiten für die Unternehmen erhöhen und dadurch die Prägekraft des Flächentarifvertrages stärken wie auch die Tarifbindung sichern sollen. Diese Regelungen wurden vor dem Hintergrund einer hohen Tarifbindung abgeschlossen. Der Organisationsgrad der Arbeitgeberverbände nach Unternehmen liegt bei über $50 \%$ und der nach Beschäftigten nach Angaben der Industriegewerkschaft Bergbau, Chemie, Energie (IG BCE) bei knapp $90 \%$; legt man die Daten des Instituts für Arbeitsmarktund Berufsforschung (IAB) zugrunde, bewegt sich Letzterer im Jahr 2007 für Westdeutschland zwischen $81 \%$ (Bergbau und Energie) und $64 \%$ (Grundstoffverarbeitung) (Ellguth/ Kohaut 2008).

Insgesamt gibt es fünf tarifliche Regelungen, die betriebsbezogene Unterschrei- 
tungen von Tarifnormen erlauben. Sie zeichnen sich dadurch aus, dass sie Abweichungsthemen festlegen, dass sie zumeist Bandbreiten für Abweichungen definieren und dass sie Verhandlungen der Betriebsparteien sowie einen Zustimmungsvorbehalt der Tarifvertragsparteien vorsehen.

(1) Arbeitszeitkorridor: Die 1994 geltende Regelung erlaubt die Arbeitszeitverlängerung oder -verkürzung um bis zu 2,5 Stunden mit Entgeltausgleich. Erstreckt sich die Geltung der Abweichung auf größere Betriebe oder Betriebsteile, ist die Zustimmung der Tarifvertragsparteien verpflichtend.

(2) Entgeltkorridor: Die Anfang 1998 in Kraft getretene Regelung eröffnet die Möglichkeit einer Entgeltabsenkung um bis zu $10 \%$. Sie ist geknüpft an Bedingungen wie die Beschäftigungssicherung, die Verbesserung der Wettbewerbsfähigkeit oder wirtschaftliche Schwierigkeiten des Unternehmens. Der Abbau übertariflicher Leistungen soll Vorrang haben.

(3) Jahresleistung/Einmalzahlung: Die zuletzt 2002 vereinbarte Regelung lässt eine Veränderung der Höhe und des Auszahlungszeitpunktes der Jahresleistung (oder anderer Einmalzahlungen wie des Urlaubsgeldes) für das laufende Kalenderjahr zu. Auch hier sind Kürzungen wirtschaftlich $\mathrm{zu}$ begründen, und Reduzierungen übertariflicher Leistungen sollten Vorrang haben.

(4) Tarifkonkurrierende Betriebe: Nach der seit Mitte 2002 gültigen Regelung können die Betriebsparteien für einzelne Betriebe oder Betriebsteile befristet niedrigere Entgeltsätze aushandeln, sofern diese in einer Tarifkonkurrenz zu anderen Tarifverträgen stehen. Die Abweichung kann auch unbefristet vereinbart werden.

(5) Firmenbezogene Verbandstarifverträge: Ebenfalls seit Mitte 2002 existiert die Möglichkeit, eine vom Verbandstarifvertrag abweichende Tarifvereinbarung für ein Unternehmen - analog zu einem Haustarifvertrag - zu treffen. Dieses Instrument soll der Ergänzung dienen und erst nach Prüfung anderer Möglichkeiten genutzt werden. Abweichungen können zu allen tariflichen Themen vereinbart werden und sind durch die Tarifvertragsparteien abzuschlieBen.

\subsection{METALLINDUSTRIE}

In der Metallindustrie war die Durchsetzung der Öffnungsklauseln von Konflikten geprägt. Die 1993 vereinbarte Härtefallklausel kam nur durch die vorzeitige Kündigung der ostdeutschen Tarifverträge und das Drängen der Arbeitgeberverbände zustande, und die Pforzheimer Vereinbarung von 2004, in der die Begrenzung von Tarifabweichungen auf Sanierungsfälle aufgehoben wurde, ist ohne den offenen Druck der rot-grünen Bundesregierung und die Androhung gesetzlicher Öffnungsklauseln im Jahr 2003 nicht zu verstehen. Dennoch war gerade die Pforzheimer Vereinbarung auch eine Reaktion auf wachsende Probleme der Tarifpraxis in der Branche. Dazu zählten sowohl die Ausweitung der wilden Dezentralisierung (vor allem in Ostdeutschland) als auch die Intransparenz und mangelnde Kontrolle über Tarifabweichungen in Westdeutschland. Erschwerend hinzu kam eine starke Abnahme der Tarifbindung, die nach Beschäftigten zwischen 1990 und 2007 um mehr als $15 \%$ auf nur noch gut $56 \%$ zurückging.

Insgesamt existieren in der Metallindustrie drei flächentarifvertragliche Regelungen zur Tarifabweichung. Wie die Regelungen der chemischen Industrie zielen sie auf zeitlich begrenzte Abweichungen mit dem Ziel, Beschäftigung zu sichern. Im Unterschied zur chemischen Industrie zeichnen sie sich dadurch aus, dass sie keine thematischen Beschränkungen vornehmen, dass sie keine Schwellenwerte für das Ausmaß materieller Konzessionen festlegen und dass sie nicht als Betriebsvereinbarungen zu verhandeln sind, sondern den Status von ergänzenden Tarifvereinbarungen haben. Die Mitte der 1990er Jahre abgeschlossenen Tarifvereinbarungen zur Standortund Beschäftigungssicherung, die den Betriebsparteien Möglichkeiten der Arbeitszeitverkürzung ohne Lohnausgleich zur Beschäftigungssicherung bieten, werden hierbei nicht als Tarifabweichungen verstanden. Sie haben zwar den Verhandlungsspielraum für betriebliche Bündnisse erweitert, garantieren jedoch die Konstanz der Stundenentgelte. Diese Form der Arbeitszeitverkürzung bedeutet deshalb keine materielle Konzession, sondern stellt eine kollektiv vereinbarte Teilzeit dar.

(1) Härtefallklauseln: Die seit Frühjahr 1993 für die ostdeutschen Tarifgebiete gel- tenden Härtefallklauseln erlauben Tarifabweichungen durch die Tarifvertragsparteien ohne thematische Einschränkungen. Bedingung ist aber der Nachweis einer wirtschaftlichen Krisensituation des Unternehmens.

(2) Sanierungsregelungen: Seit Mitte der 1990er Jahre wurden in mehreren westdeutschen Tarifgebieten Öffnungsklauseln vereinbart, die unternehmensbezogene $\mathrm{Ab}$ weichungen bei wirtschaftlichen Schwierigkeiten erlaubten. Teilweise waren diese Regelungen auf bestimmte Themen - wie die Verschiebung von Entgelterhöhungen beschränkt, teilweise waren sie auch thematisch offen.

(3) Pforzheimer Vereinbarung: In der Pforzheimer Vereinbarung des Jahres 2004 wird, wie bereits erwähnt, die Begrenzung auf Sanierungsfälle aufgehoben. Als Bedingung für den Abschluss einer Tarifabweichung gelten die Sicherung oder der Ausbau der Beschäftigung sowie die Verbesserung der Innovations- und Wettbewerbsfähigkeit und der Investitionsbedingungen. Die Vereinbarung sieht dafür die Prüfung von Maßnahmen durch die Betriebsparteien unter Hinzuziehung der Tarifvertragsparteien vor. Die Unternehmen sind zu einer umfassenden Information und zur Bereitstellung entsprechender Unterlagen verpflichtet.

\section{Anzahl und Inhalte der Tarifabweichung}

Die Datenlage zur Verbreitung von Tarifabweichungen und ihren Inhalten ist für die beiden Branchen unterschiedlich. Für die chemische Industrie liegen Daten der Gewerkschaft und des Arbeitgeberverbandes vor, die jeweils den gesamten Zeitraum seit Vereinbarung der entsprechenden Tarifregelungen bis 2007 abdecken. Allerdings bezieht sich diese Dokumentation nur auf die Verbreitung der einzelnen Instrumente und nicht auf die Anzahl abweichender Vereinbarungen. Auch existieren keine weiteren Informationen über die Inhalte der Abweichungen oder die Gegenleistungen der Unternehmen. In der Metallindustrie werden die abweichenden Vereinbarungen seit 2004 sowohl in den Bezirken als 
auch im zentralen Tarifarchiv erfasst und dort nach bestimmten Kategorien ausgewertet. $^{2}$

\subsection{CHEMISCHE INDUSTRIE}

In der chemischen Industrie waren die Anteile der Instrumente zur Tarifabweichung Ende 2006 wie folgt verteilt: Die am meisten genutzten Instrumente waren der Arbeitszeitkorridor (41 \% Anteil an allen Nutzungen) und der Entgeltkorridor (33\%). Mehr als jede fünfte Nutzung entfällt auf die firmenbezogenen Verbandstarifverträge $(22 \%)$. Die Einmalzahlung rangiert mit einem Anteil von $13 \%$ auf dem vierten Platz. Tarifkonkurrierende Regelungen, beispielsweise für Dienstleistungsbereiche der Unternehmen, spielen nur eine untergeordnete Rolle (1\%).

Aus den Interviews und Fallstudien ist bekannt, dass die Abweichungsinstrumente zumeist gemeinsam genutzt werden. Besonders häufig findet sich demnach die Verbindung von Arbeitszeitverlängerung und Entgeltkürzung. Unter den Arbeitszeitabweichungen dominiert die Arbeitszeitverlängerung, die 2006 einen Anteil von fast $80 \%$ aufwies, und darunter sahen fast drei Viertel der Fälle eine Verlängerung von 37,5 auf 40 Wochenstunden vor. Zu den Inhalten der firmenbezogenen Verbandstarifverträge wie auch der tarifkonkurrierenden Regelungen liegen keine Informationen vor. In diesen Vereinbarungen sind mit hoher Wahrscheinlichkeit die Grenzen der Korridore für materielle Konzessionen überschritten worden. Das Muster der zeitlichen Dynamik der Tarifabweichung ist für die Korridorregelungen und die Jahresleistung - nur zu diesen Instrumenten existieren Zahlen - ähnlich: Einer zunächst geringen Nutzung folgt im Jahr 2004 ein starker Anstieg, der seinen Scheitelpunkt 2005 erreicht und dann wieder zurückgeht ( $A b$ bildung 1).

\subsection{METALLINDUSTRIE}

In der Metallindustrie ähnelt die zeitliche Dynamik der Tarifabweichung stark der in der chemischen Industrie. Auch hier schnellte die Anzahl der Tarifabweichungen im Jahr 2005 hoch, um dann 2006 wieder deutlich abzusinken. Von den im Zeitraum von 2004 bis 2006 abgeschlossenen insgesamt 850 abweichenden Vereinbarungen entfielen $48 \%$ auf das Jahr 2005, aber nur $32 \%$ auf 2006 und $20 \%$ auf 2004. Die

\section{Abb. 1: Erteilte Genehmigungen für Arbeitszeitkorridor, Entgeltkorridor und die Jahresleistung*}

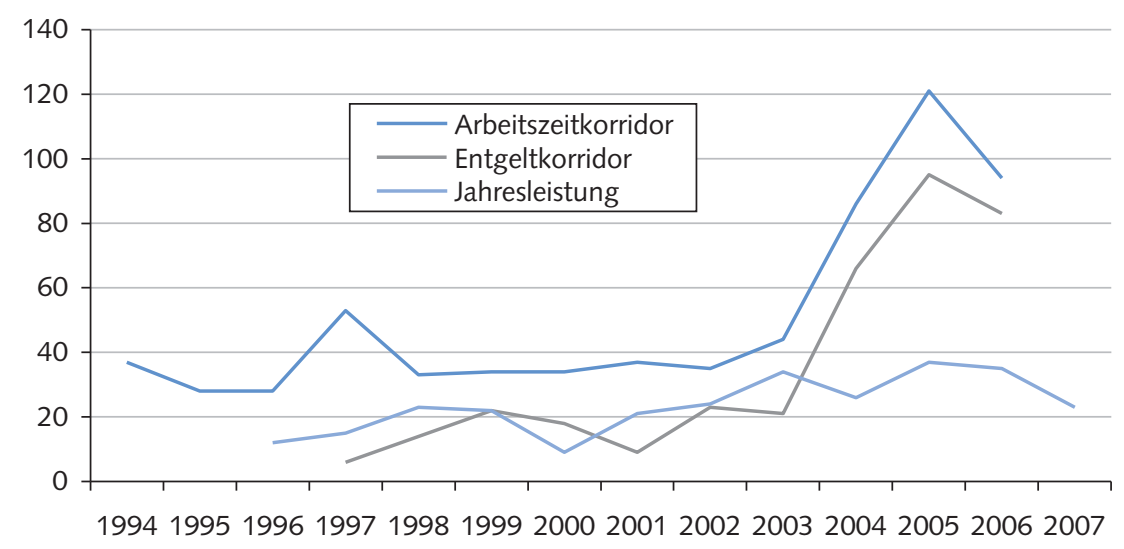

*ausschließlich Westdeutschland. Quelle: IG BCE.

WSI MiTTEILUNGEN

Abweichungsquote bezogen auf alle tarifgebundenen Unternehmen der Branche liegt 2006 bei gut $10 \%$. Ähnlich lautet auch eine gewerkschaftliche Schätzung für die chemische Industrie (Förster 2008).

Anders als in der chemischen Industrie sind in der Metallindustrie Arbeitszeit und Entgelt als wichtigste Konzessionsthemen etwa gleich verbreitet. Die Anteile von jeweils knapp $70 \%$ an allen abweichenden Vereinbarungen für beide Themen implizieren zudem, dass die Themen häufig auch kombiniert auftreten. Von wachsender Bedeutung ist das Thema der neuen Entgeltrahmenabkommen (ERA), dessen Anteile an den abweichenden Vereinbarungen sich im Untersuchungszeitraum mehr als verdoppelt haben. 2006 enthielt fast jede zweite Vereinbarung eine Abweichung zum Thema ERA, was zum einen an den nahenden ERA-Einführungsterminen lag und zum anderen daran, dass sich ERA für Kostensenkungen aus Sicht der Gewerkschaft gut eignete, weil damit nicht spürbar in die tariflichen Entgelte eingegriffen wurde.

Das wichtigste Einzelthema der materiellen Konzessionen aber war die Arbeitszeitverlängerung ohne Entgeltausgleich, die in gut $58 \%$ aller Vereinbarungen enthalten war. $60 \%$ darunter bestanden wiederum aus Verlängerungen der Wochenarbeitszeit. Andere Formen waren zusätzliche Arbeitszeitkontingente oder Qualifizierungszeiten, deren Anteil im Untersuchungszeitraum stark anstieg. Dies ist ebenso als Hinweis auf einen Kontrollanstieg der Gewerkschaft zu werten wie die Zunahme von Rückkehrregelungen, in denen verbindliche Schritte zur Rückkehr auf das Flächentarifvertragsniveau bereits während der Laufzeit der Vereinbarung definiert werden. Ihr Anteil an den vereinbarten Wochenarbeitszeitverlängerungen betrug 2006 fast $30 \%$.

Insbesondere bei den Gegenleistungen der Unternehmen sind Verhandlungserfolge der Gewerkschaft unübersehbar. Alle in den Tarifabweichungen ausgehandelten Gegenleistungen - mit Ausnahme von Bonusregelungen, die besondere Leistungen für Gewerkschaftsmitglieder definieren -, weisen stark steigende Anteile auf (Abbildung 2). Inzwischen gibt es kaum mehr eine Tarifabweichung, die keine Beschäftigungssicherung enthält. Hoch einzuschätzen ist der Anstieg der Investitionszusagen, die zumindest potenziell eine Verbesserung der Wettbewerbsfähigkeit nach sich ziehen können und es den Unternehmen dann auch ermöglichen würden, nach Ablauf der abweichenden Vereinbarung wieder zu den Normen der Flächentarifverträge zurückzukehren. Damit ist freilich keine Garantie verbunden; doch immerhin konnte die durchschnittliche Investitionssumme pro Vereinbarung im Untersuchungszeitraum vervielfacht werden. $\mathrm{Zu}$ einer geordneten

\footnotetext{
2 In diesem Zusammenhang kann auch auf eine Analyse der Tarifabweichungen nach dem Pforzheimer Abkommen zurückgegriffen werden, die ich durchgeführt habe und die Aussagen zur Anzahl, zu materiellen Konzessionen, Gegenleistungen und Verfahrensregelungen der Vereinbarungen zwischen 2004 und 2006 ermöglicht. Dass sich keine Angaben zur Verbreitung der Tarifabweichung vor 2004 machen lassen, war ein gewichtiger Teil des Problems, das schließlich auch zum Pforzheimer Tarifvertrag geführt hat.
} 
Abb. 2: In Tarifabweichungen ausgehandelte Gegenleistungen - Anteile der Themen in \% -

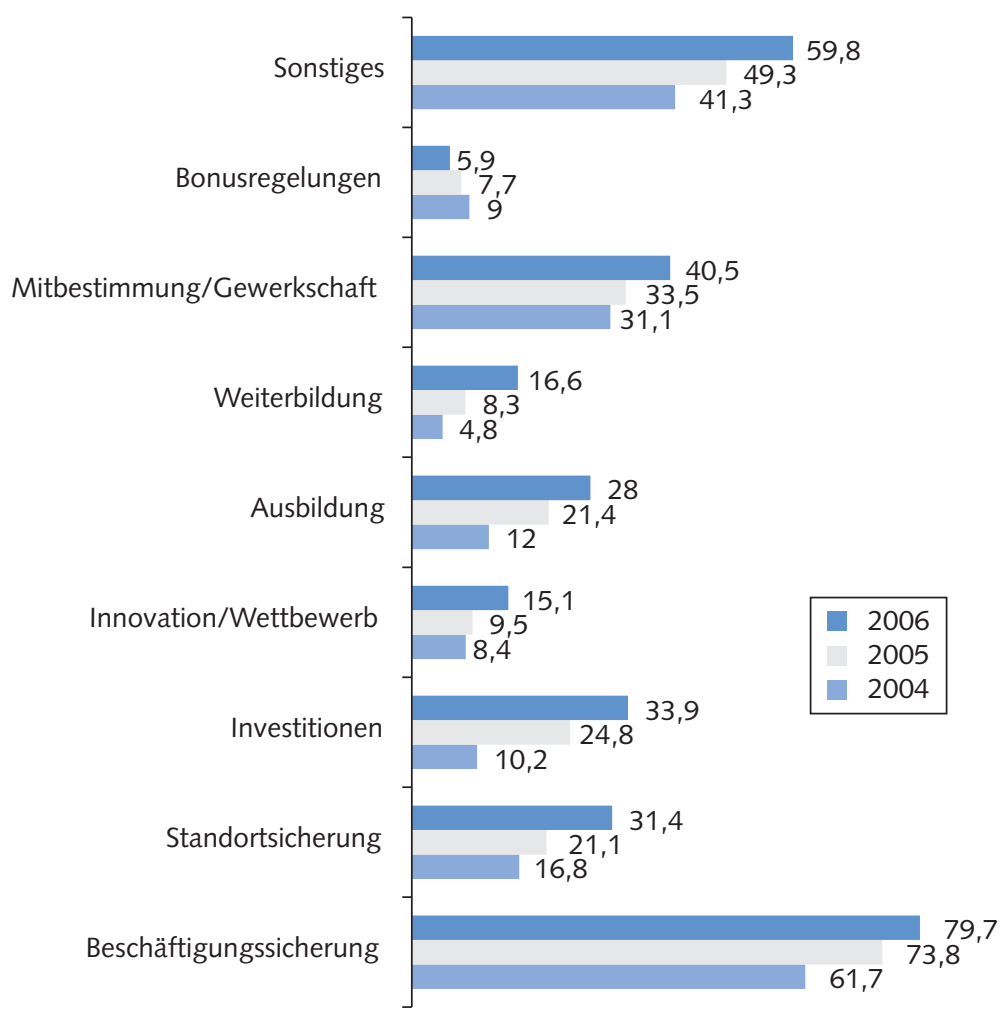

Quelle: Eigene Auswertungen des Autors.

WSI MITTELIUNGEN

Rückkehr beitragen können auch die verbesserten Mitbestimmungsrechte der Arbeitnehmervertreter, hinter denen sich vor allem Kontrollrechte bei der Umsetzung der Tarifabweichung verbergen.

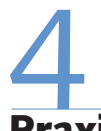

Praxis der Tarifabweichung: Artikulation und Aushandlung

In beiden Branchen war die Ausweitung der Tarifabweichung seit 2004 zunächst von gravierenden Kontrollproblemen begleitet. Die Gewerkschaften haben hierauf jeweils sehr ähnlich reagiert. Sowohl die IG BCE als auch die IG Metall haben versucht, die Prozesskontrolle durch Zentralisierung zu verbessern, um auf diese Weise auch die Verbreitung und die Inhalte von Tarifabweichungen besser kontrollieren zu können. Dies ist ihnen auch in einem bemerkenswerten Umfang gelungen. Nichtsdestotrotz zeigen sich dabei auch deutliche Unterschiede in den lokalen Verhandlungsprozessen.

\subsection{DIE ARTIKULATION ZWISCHEN DEN VERBANDSEBENEN}

Das Artikulationsmuster zwischen den Verbandsebenen beider Gewerkschaften hat sich erstaunlich gleichartig entwickelt. Zunächst war es in beiden Branchen so, die Gewerkschaften keine größere Bedeutung hatte. Es herrschte die Erwartung vor, dass die jeweiligen Regelungen im Flächentarifvertrag genügten, um die Aushandlung von Tarifabweichungen zu steuern. In der chemischen Industrie lag dabei die Handlungsinitiative ganz bei den Betriebsparteien. In vielen Fällen wurden Vereinbarungen betrieblich verhandelt und dann der Gewerkschaft zur Unterschrift zugeschickt, was diese zumeist - akzeptierte. In der Metallindustrie wiederum sollten die in der Gewerkschaft wahrgenommenen Probleme der Sanierungspraxis gerade durch die Pforzheimer Vereinbarung angegangen der wirtschaftlichen Situation und eine aktive Rolle der Tarifvertragsparteien vorsah. dass die Frage der Prozesskontrolle für werden, die verpflichtende Nachweise
In beiden Branchen aber brachen mit dem Anstieg der Tarifabweichungen zwischen 2004 und 2005 Kontrollprobleme auf. Immer mehr Unternehmen drängten auf Tarifabweichungen, in der Metallindustrie nicht zuletzt wegen der Vereinbarung von Pforzheim, in der chemischen Industrie aber auch unabhängig von neuen tariflichen Regelungen. Arbeitszeitverlängerungen ohne Entgeltausgleich standen dabei im Zentrum.

Diese neue Dynamik der Tarifabweichungen wurde von beiden Gewerkschaften schließlich als Bedrohung für die Prägekraft der Flächentarifverträge gewertet. In der chemischen Industrie häuften sich in den Jahren 2004 und 2005 Fälle, bei denen den Betriebsräten Tarifabweichungen durch Drohungen wie Standortverlagerungen oder Outsourcing auch in wirtschaftlich erfolgreichen Unternehmen abgerungen wurden oder sogar, wie im Falle eines Reifenstandortes von Continental, bereits getroffene Vereinbarungen von Unternehmensleitungen nicht eingehalten worden waren. In dieser Situation wurde im Verlauf des Jahres 2005 vom Vorstand der Gewerkschaft eine so bezeichnete ,interne Verabredung" mit dem Ziel entwickelt, die prozedurale Kontrolle deutlich zu verbessern.

Das darin skizzierte idealtypische Verfahren sah folgende Stufen vor: Die Gewerkschaft ist über eine Forderung der Unternehmen nach Tarifabweichungen umgehend zu informieren; die Tarifabteilung im Vorstand entscheidet zentral nach Prüfung der wirtschaftlichen Ausgangsbedingungen über den Beginn von Verhandlungen; Verhandlungen im Betrieb finden nur mit Teilnahme eines gewerkschaftlichen Vertreters (in kleineren Unternehmen von Bezirk oder Landesbezirk, in größeren Unternehmen auch ein Tarifsekretär der Tarifabteilung) statt; die Verhandlungen und Ergebnisse sind in Mitgliederversammlungen zu kommunizieren, und schließlich bedarf eine Vereinbarung der Genehmigung durch die Tarifabteilung. Inhaltliche Kriterien für Ablehnungen wurden nicht formuliert. Allerdings sollten Tarifabweichungen, die nur dem Ziel der Renditesteigerung dienen, und Vereinbarungen, die absehbar Abweichungswünsche bei Konkurrenten oder konkurrierenden Standorten nach sich ziehen, nicht genehmigt werden. Neu war auch die Empfehlung, die Mitglieder der Gewerkschaft privilegiert zu informieren, ein Punkt, der zuvor in den 
Überlegungen der Gewerkschaft gar keine Rolle gespielt hatte.

In der Metallindustrie hatte die Pforzheimer Vereinbarung zunächst den Effekt, dass Kontrollprobleme offen zu Tage traten, die zuvor durch den Schleier der Intransparenz verdeckt worden waren. Wie in der chemischen Industrie mehrten sich in den Jahren 2004 und 2005 Fälle, in denen Betriebsräte ohne Beteiligung der Gewerkschaft weitgehende materielle Konzessionen - insbesondere der Arbeitszeitverlängerung ohne Entgeltausgleich - zusagten, die von der Gewerkschaft dann in den Verhandlungen kaum mehr rückgängig gemacht werden konnten. Auch die IG Metall hat deshalb im Jahr 2005 , Koordinierungsregeln" für die Aushandlung von Abweichungen entwickelt. Dazu gehörten folgende Punkte: Anträge auf Verhandlung von Abweichungen sind an die Bezirksleitungen zu stellen und von diesen auf der Grundlage umfassender Informationen des Betriebes zu entscheiden; Bezirksleitungen können die Verwaltungsstellen zu Verhandlungen ermächtigen; Verhandlungen sind von betrieblichen Tarifkommissionen zu begleiten, die eine Beteiligung der Mitglieder sicherstellen sollen; Verhandlungsergebnisse sind schließlich an den Vorstand weiterzuleiten, der sie zu genehmigen und zu verantworten hat. Ein wichtiges Kriterium dafür war, solche Tarifabweichungen nicht zuzulassen, die sich Wettbewerbsvorteile durch Abwertungsspiralen der Arbeitsstandards verschaffen wollen.

Die Regelungen beider Branchen ähneln sich stark. In beiden Fällen geht es um die zentrale Kontrolle von Informationsund Entscheidungsflüssen sowohl bei der Zustimmung zu Verhandlungen als auch zu den Verhandlungsergebnissen. Nach Aussage von Vertretern beider Tarifparteien in den Branchen und nach den Fallstudienerfahrungen im oben angesprochenen Forschungsprojekt haben sich die neuen Verfahrensrichtlinien bei Tarifabweichungen mittlerweile in beiden Branchen fest etabliert. Abweichungen werden zentral angekündigt, es finden kaum mehr Verhandlungen ohne Beteiligung der Tarifparteien statt, und die Tarifparteien haben das letzte Wort bei der Entscheidung über Annahme oder Ablehnung eines Verhandlungsergebnisses. Freilich gilt dies nur für formelle Tarifabweichungen und nicht für die wilde Dezentralisierung. Zumindest für die Metallindustrie aber lässt sich nach
Aussage von Tarifexperten beider Tarifparteien sagen, dass mit der Pforzheimer Vereinbarung und den neuen Verfahrensrichtlinien auch das Ausmaß der wilden Dezentralisierung-zumindest bislang - rückläufig ist. In der chemischen Industrie hatte dieses Problem keine solche Virulenz.

Der wichtigste Unterschied zwischen beiden Branchen besteht im Partizipationsgehalt der Verfahren. In der chemischen Industrie wird die Information der Mitglieder in Mitgliederversammlungen zwar empfohlen, doch eine systematische Beteiligung ist damit nicht verbunden. In der Metallindustrie hingegen soll über die Bildung von betrieblichen Tarifkommissionen und über Mitgliederentscheidungen die Mitgliederbeteiligung zu einem grundlegenden Bestandteil der Verfahren gemacht werden. Die Beteiligung soll nicht nur dem Ziel dienen, die Kontrolle über die Prozesse der Tarifabweichung zu verbessern, ${ }^{3}$ sondern sie intendiert ausdrücklich, die gewerkschaftliche Organisationsmacht im Betrieb zu stärken (Huber et al. 2006). Durch bevorzugte Partizipation der Mitglieder an Verhandlungen und Entscheidungen zur Tarifabweichung soll die Mitgliedernähe der Gewerkschaft verbessert und damit ihre Attraktivität für Mitglieder - vorhandene und potenzielle - erhöht werden. Welche Prägekraft aber haben diese Verfahrensvorgaben tatsächlich für die soziale Praxis im Betrieb?

\subsection{LOKALE AUSHANDLUNGEN UND AKTEURE}

Die Aushandlungspraxis ist auf den ersten Blick vielfältig. In beiden Branchen finden sich in Fallstudien Beispiele, in denen die Kontrollvorgaben in problematischer Weise gehandhabt werden. In der chemischen Industrie bestehen die Probleme vor allem darin, dass die Betriebsparteien vor Einbeziehung der Gewerkschaft Vorabsprachen getroffen haben. Auch Mitgliederversammlungen sind keine gängige Praxis. In der Metallindustrie ist das zentrale Problem, dass lokale Verhandlungsakteure die Beteiligungsverfahren nur unzureichend umsetzen. In diesen Fällen werden die Tarifkommissionen auf wenige Personen beschränkt und von Betriebsrat und Betreuern bestimmt, anstatt von den Mitgliedern gewählt zu werden, oder es wird auf eine Entscheidung der Mitglieder über die Annahme eines Verhandlungsergebnisses verzichtet.
Die auftretenden Probleme sind bei aller Unterschiedlichkeit nicht zufällig. So zeigen sich jeweils deutliche Bezüge zu den unterschiedlichen Regelungsgrundlagen. Weil Abweichungen den Status von Betriebsvereinbarungen haben, finden sich Probleme in der chemischen Industrie vor allem an der Schnittstelle zwischen den Betriebs- und den Tarifparteien. Auch überrascht es nicht, dass dort die Mitgliederinformation nur eine untergeordnete praktische Bedeutung hat. In der Metallindustrie ist die leitende Rolle der Tarifpartei Gewerkschaft bei den Verhandlungen weit weniger umstritten; sie ist Herrin des Verfahrens, weil die Tarifabweichung als Tarifvereinbarung verhandelt wird. Hier entstehen die Probleme vorrangig aus Umsetzungsdefiziten der neuartigen Beteiligungsverfahren, die für die lokalen Interessenvertretungen zwangsläufig mit einem neuen Rollenverständnis verbunden sind, weil sie sich der Diskussion und vor allem den Entscheidungen der Mitglieder stellen müssen.

Trotz dieser Probleme zeigen andere Fälle in der Metallindustrie, dass bei erfolgreicher Umsetzung der beteiligungsorientierten Verfahren ein Legitimationsgewinn für die Interessenvertretungen entstehen kann. Dies ist ein entscheidender Vorzug solcher Verfahren. Auch in der chemischen Industrie gibt es Fälle, in denen die Beschäftigten gut informiert und eingebunden werden. Insbesondere dort, wo die Interessenvertretungen konfliktorientierte Strategien entwickelt haben und die Beschäftigten mobilisiert wurden, haben die Verhandlungen zu Tarifabweichungen die betriebliche Stellung der Interessenvertretungen gestärkt. Allerdings wird dabei von den Beschäftigten selten zwischen Betriebsrat und Gewerkschaft unterschieden, sodass eine Steigerung der gewerkschaftlichen Organisationsmacht zumeist nicht erfolgt. Die Vermeidung von Mitgliederverlusten wird hier schon als Erfolg gewertet. Dies ist in der Metallindustrie anders, wo wenn auch nur in vereinzelten Fällen - die Stellung der Gewerkschaft im Betrieb gefestigt wurde oder sogar starke Mitgliedergewinne verzeichnet werden konnten. Die notwendige Bedingung dafür scheint $\mathrm{zu}$

3 Hier wären sogar mögliche Widersprüche zwischen zentraler Kontrolle und dezentralen Beteiligungsverfahren nicht auszuschließen, z. B. wenn Vorstand und Mitglieder ein Verhandlungsergebnis nicht einheitlich bewerten 
sein, dass die Beteiligungsverfahren ernsthaft und mit dem ausdrücklichen Ziel der Mitgliedergewinnung umgesetzt werden. Die Legitimationsfrage ist für die Interessenvertretungen vor allem deshalb so bedeutend, weil in allen untersuchten Fällen die Belegschaften einer Tarifabweichung zunächst mehrheitlich skeptisch gegenüberstanden und sich deshalb die Interessenvertretungen für Tarifabweichungen erst eine Legitimationsbasis schaffen mussten.

Allerdings hat die Ausrichtung auf beteiligungsorientierte Verfahren für die Gewerkschaften auch einen Preis. Diese sind nämlich sinnvoll nur dort anzuwenden, wo die Gewerkschaften ein Mindestmaß an Organisationskraft haben, die sie stärken und in den Verhandlungen mit den Unternehmen nutzen können. Deshalb entscheidet sich die IG Metall nach Aussage befragter Gewerkschaftssekretäre zunehmend, Abweichungen nur zu verhandeln, falls eine gewerkschaftliche Mindeststärke bereits besteht oder der Betriebsrat und auch die Unternehmensseite die Mitgliederrekrutierung im Vorfeld der Verhandlungen unterstützen.

\section{5 \\ Bewertung}

Tarifabweichungen sind zunächst als eine Gefährdung für die Prägekraft der Flächentarifverträge einzustufen, weil sie auf der Ebene der Einzelunternehmen Tarifnormen unterschreiten und dadurch Arbeits- standards definieren, die mit den Tarifnormen konkurrieren. Zugleich liegt die Verhandlungsinitiative bei den Unternehmen. Gewerkschaften und Betriebsräte handeln aus der Defensive. Diese Defensive ist aber keine Folge der Tarifabweichungen, sondern den Machtverschiebungen zugunsten der Unternehmen geschuldet, die nun einen neuartigen Druck auf Tarifnormen ausüben können. Aus den Erfahrungen der chemischen und der Metallindustrie lässt sich folgern, dass in dieser Situation Tarifabweichungen den Interessenvertretungen zumindest potenziell Möglichkeiten bieten, die Probleme, die sich aus den Machtverschiebungen für die Tarifregulierung ergeben, aktiv anzugehen. Dafür lassen sich zwei Argumente anführen.

Erstens führen Tarifabweichungen nicht per se zu einer Erosion der Flächentarifverträge. Ihre Auswirkungen auf die Flächentarifverträge hängen vielmehr entscheidend davon ab, wie gut die Gewerkschaften mit dem Problem in der Praxis umgehen. Sofern die Gewerkschaften die inhaltliche und prozedurale Kontrolle von Tarifabweichungen zum Problem ihrer Organisation machen und aktiv verfolgen, bestehen durchaus Chancen, Anzahl und Reichweite materieller Konzessionen einzudämmen und erfolgreich Gegenleistungen der Unternehmen einzufordern. Nicht von ungefähr stagniert die Abweichungsquote in beiden Branchen bei etwa $10 \%$; einen Flächenbrand der Tarifabweichung gab es damit bislang nicht. Zudem lässt sich sagen, dass beteiligungsorientierte Verfahren Möglichkeiten einer neuen Legitimierung gewerkschaftlichen Handelns bieten, die ei- ne Festigung der gewerkschaftlichen Organisationsmacht im Betrieb nach sich ziehen können. Auf diese Weise bietet sich sogar die Chance, dass die Gewerkschaften ein Stück aus ihrer strukturellen Defensive herauskommen.

Zweitens ist zu bedenken, dass die Tarifabweichungen nicht die Ursache der abnehmenden Prägekraft der Tarifverträge, sondern ihre Folge sind. Anders als die wilde Dezentralisierung bieten Tarifabweichungen immerhin Ansatzpunkte für gewerkschaftliche Kontrollpraktiken. Sofern diese auch genutzt werden, können Tarifabweichungen sich zu wirkungsvollen Instrumenten entwickeln, die zur Eindämmung der Erosion des Flächentarifvertrages beitragen. Nicht von ungefähr ist in der chemischen Industrie die Tarifbindung konstant hoch und konnte in der Metallindustrie die wilde Erosion - zumindest nach allem, was wir wissen - begrenzt werden.

Freilich gilt bei dieser Bewertung, dass die Gewerkschaften für eine erfolgreiche Kontrollpraxis ein Mindestmaß an Durchsetzungskraft und betrieblicher Präsenz haben müssen. Wo diese Bedingungen nicht gegeben sind, geraten die Möglichkeiten einer offensiven gewerkschaftlichen Strategie im Umgang mit Tarifabweichungen zwangsläufig an Grenzen. In der Folge könnte die Differenzierung von Tarifstandards zwischen Betrieben, aber auch zwischen Branchen, abhängig von der Organisationsmacht der Gewerkschaft weiter zunehmen. Aber auch hier gilt, dass ohne die Tarifabweichung die Probleme kaum kleiner wären; sie wären nur weniger transparent. 


\section{LITERATUR}

Bispinck, R. (2004a): Tarifliche Öffnungsklauseln. Eine Analyse von rund 30 Tarifbereichen, Düsseldorf

Bispinck, R. (2004b): Kontrollierte Dezentralisierung der Tarifpolitik Eine schwierige Balance, in: WSI-Mitteilungen 5, S. 237-245

Crouch, C. (1993): Industrial Relations and European State Traditions, Oxford

Ellguth, P./Kohaut, S. (2008): Tarifbindung und betriebliche Interessenvertretung: Aktuelle Ergebnisse aus dem IAB-Betriebspanel 2007, in: WSIMittelungen 9, S. 515-519

Förster, G. (2008): Stabilisierung des Flächentarifvertrages durch Flexibilisierung. Tarifpolitische Konzepte und Erfahrungen der IG BCE, in:

Bispinck, R. (Hrsg.): Verteilungskämpfe und Modernisierung, Hamburg, S. $151-158$

Hassel, A. (1999): The Erosion of the German System of Industrial Relations, in: British Journal of Industrial Relations 37, S. 483-505
Huber, B./Burkhard, O./Wagner, H. (Hrsg.) (2006): Perspektiven der Tarifpolitik. Im Spannungsfeld von Fläche und Betrieb, Hamburg Jacobi, O. (2003): Renewal of the Collective Bargaining System?, in: Müller-Jentsch, W./Weitbrecht, H. (Hrsg.): The Changing Contours of German Industrial Relations, München und Mering, S. 15-38 Massa-Wirth, H. (2007): Zugeständnisse für Arbeitsplätze? Konzessionäre Beschäftigungsvereinbarungen im Vergleich Deutschland - USA, Berlin Rehder, B. (2003): Betriebliche Bündnisse für Arbeit in Deutschland. Mitbestimmung und Flächentarif im Wandel, Frankfurt/Main und New York Seifert, H./Massa-Wirth, H. (2005): Pacts for employment and competitiveness in Germany, in: Industrial Relations Journal 3, S. 217-240 Traxler, F. (1995): Farewell to Labour Market Associations? Organized versus Disorganized Decentralization as a Map for Industrial Relations, in: Traxler, F./Crouch, C. (Hrsg.): Organized Industrial Relations in Europe: What Future?, Aldershot, S. 3-21 